[0212-7199 (2006) 23: 5; pp 206-212] ANALES DE MEDICINA INTERNA Copyright (C) 2006 ARAN EDICIONES, S.L

AN. MED. INTERNA (Madrid) Vol. 23, N. ${ }^{\circ}$ 5, pp. 206-212, 2006

\title{
Prevalencia de hemoglobinopatías en mujeres gestantes en el área sanitaria de Lanzarote
}

\author{
J. M. CALVO-VILLAS, M. F. ZAPATA RAMOS ${ }^{1}$, J. CUESTA TOVAR, S. DE LA \\ IGLESIA ÍNIGO ${ }^{2}$, P. ROPERO GRADILLA ${ }^{3}$, E. CARRETER DE GRANDA, \\ F. SICILIA GUILLÉN
}

Servicios de Hematología y Hemoterapia $y^{\prime}$ Análisis Clínicos. Hospital General de Lanzarote. ${ }^{2}$ Servicio de Hematología. Hospital Doctor Negrín. Las Palmas. ${ }^{3}$ Servicio de Hematología. Hospital Clínico Universitario San Carlos. Madrid

\begin{abstract}
PREVALENCE OF HEMOGLOBINOPATHIES IN PREGNANT WOMEN IN THE LANZAROTE HEALTH SANITARY AREA
\end{abstract}

\section{RESUMEN}

Fundamento: El objetivo de este trabajo es el estudio de la prevalencia de las hemoglobinopatías heterocigotas en las mujeres gestantes residentes en Lanzarote.

Pacientes y métodos: Se ha puesto en marcha un estudio epidemiológico observacional transversal para determinar la prevalencia de hemoglobinopatías en 2.436 mujeres gestantes en Lanzarote. El método diagnóstico de despistaje para las hemoglobinas variantes fue la electroforesis de hemoglobinas en acetato de celulosa a $\mathrm{pH}$ alcalino y para la $\beta$ talasemia la cuantificación de hemoglobinas $\mathrm{A}_{2}$ y fetal. El estudio de confirmación de una hemoglobinopatía estructural se basó en la electroforesis de hemoglobinas en agar citrato a $\mathrm{pH}$ ácido, el isoelectroenfoque y la cromatografía líquida de alta resolución (HPLC). El estudio molecular de la $\beta$ talasemia $\left(\mathrm{HbA}_{2}>3,5 \%\right)$ se realizó con técnicas de PCR en tiempo real y sondas marcadas con fluorógenos y la PCR con amplificación de alelos específicos (PCR-ARMS).

Resultados: La prevalencia global de portadores de hemoglobinopatías fue $11,90 \%$, de los que $9,44 \%$ eran hemoglobinopatías estructurales y $2,46 \%$ o $\beta$ talasemias heterocigotas. Se detectó una hemoglobina variante en 23 mujeres y la distribución fue: trece casos con hemoglobinas $\mathrm{S}$, siete portadoras de $\mathrm{HbC}$, dos de $\mathrm{HbD}$ y una hemoglobina inestable. El $82,6 \%$ de las hemoglobinas variantes correspondían a población inmigrante con origen en Africa y América.

Conclusiones: La alta prevalencia de portadores de hemoglobinopatías estructurales en Lanzarote justifica la puesta en marcha de programas prospectivos de detección de portadores para prevenir la aparición de las formas severas de la enfermedad.

PALABRAS CLAVE: Hemoglobinopatías. Mujeres gestantes. Lanzarote.

Calvo-Villas JM, Zapata Ramos MF, Cuesta Tovar J, De la Iglesia Iñigo S, Ropero Gradilla P, Carreter de Granda E, Sicilia Guillén F. Prevalencia de hemoglobinopatías en mujeres gestantes en el área sanitaria de Lanzarote. An Med Interna (Madrid) $2006 ; 23: 206-212$.

\begin{abstract}
Background: The aim of this study is to analyze the global prevalence of carriers of heterozygous hemoglobinopathies among pregnant women settled in Lanzarote.

Patients and methods: A epidemiologic cross-sectional observational investigation was undertaken to study the prevalence of hemoglobinopathies in 2,436 pregnant women in Lanzarote. The techniques of primary screening were hemoglobin electrophoresis on cellulose acetate at alkaline $\mathrm{pH}$ for the detection of hemoglobin variants, and the quantification of $\mathrm{HbA}_{2}$ and $\mathrm{HbF}$ for the diagnosis of $\beta$ thalassemia trait. The study to confirm the diagnosis of structural hemoglobinopathies was based on hemoglobin electrophoresis on citrate agar at acid $\mathrm{pH}$, isolectric focusing and high-performance liquid chromatography (HPLC). The molecular characterization of $\beta$ thalassemia trait $\left(\mathrm{HbA}_{2}>3.5 \%\right)$ was carried out by techniques using a real time PCR procedure with specific fluorescently labelled hybridization probes and allele-specific amplification (PCR-ARMS).

Results: The global prevalence of hemoglobinopathies was $11.90 \%$ corresponding to $9.44 \%$ o for structural hemoglobinopathies and $2.46 \%$ for heterozygous $\beta$ thalassemias. A variant hemoglobin was detected on 23 women and the distribution was as follows: thirteen carriers of hemoglobin $S$, seven HbC trait, two HbD trait and one "unstable" hemoglobin. $82.6 \%$ of the variant hemoglobins found were from migrant population from Africa and America.

Conclusions: The high prevalence of carriers of structural hemoglobinopathies in Lanzarote justifies the initation of programs for screening for hemoglobinopathies to prevent the emergence of severe states causing disease.
\end{abstract}

KEY WORDS: Hemoglobinopathies. Pregnant women. Lanzarote.

\section{INTRODUCCIÓN}

Las hemoglobinopatías son trastornos congénitos de la hemoglobina con un patrón de herencia autosómico recesivo que incluyen las hemoglobinopatías estructurales producidas por la síntesis de una cadena de globina estructuralmente anormal, y los síndromes talasémicos que se manifiestan por la disminución total o parcial de la síntesis de una globina de estructura normal (1). Las alteraciones hereditarias de la hemoglobina constituyen un problema de salud pública en

Trabajo aceptado: 13 de diciembre de 2005

Correspondencia: José Manuel Calvo Villas. Servicio de Hematología. Hospital General de Lanzarote. Ctra. de Arrecife-Tinajo km 1,300. 35500 Arrecife de Lanzarote. Las Palmas. e-mail: jmcvillas@hotmail.com 
diferentes áreas geográficas repartidas por todo el mundo, en la mayoría de las ocasiones en el estado de portador cursan de forma silente o asintomática pero en su forma homocigota o doble heterocigota pueden causar una enfermedad grave.

El Grupo de Estudio de Hemoglobinopatías y Talasemias (GEHBTA) en 1986 recogió las tasas de prevalencia de la mayoría de regiones españolas, con una distribución heterogénea de la $\beta$ talasemia heterocigota entre las diferentes autonomías (2). La prevalencia global promedio en España se ha estimado en el $0,4 \%$, que corresponde a un caso de $\beta$ talasemia heterocigota por cada 250 habitantes (3), con una distribución que va desde su presencia testimonial en el País Vasco hasta alcanzar el 2,67\% en la isla de Menorca (4).

España no dispone en la actualidad de un registro nacional oficial de las hemoglobinopatías estructurales en niños ni en adultos. Las tasas de prevalencia han mostrado ligeras diferencias en las series publicadas con una distribución irregular que varía entre el 0,138\% de Barcelona (5) hasta el 1,17\% en neonatos de la comunidad de Madrid (6) que es la mayor tasa de prevalencia descrita en la población residente en España, ligeramente superior a la registrada en individuos gitanos de Granada que fue del 0,965\% (7).

En la Comunidad Canaria un estudio en adultos de origen hindú residentes en Gran Canaria mostró una prevalencia de $\beta$ talasemia del 10,5\% (8), la mayor tasa registrada hasta el momento en España. La tasa de prevalencia de las hemoglobinopatías estructurales en Gran Canaria fue 0,27\% (9), superior a los resultados publicados en diferentes zonas del territorio español hasta el año 1992 (9). La mayor incidencia de hemoglobinopatías en Canarias se atribuyó a la mezcla de inmigrantes europeos originarios de España, Portugal, Italia y Francia con una población nativa de origen desconocido (guanches) y con un gran número de individuos africanos de raza negra que llegaron como esclavos en los siglos XVI y XVII al archipiélago (9).

Los datos publicados sobre hemoglobinopatías en Canarias $(8,9)$, y el aumento progresivo de la inmigración en los últimos años desde países con altas tasas de hemoglobinopatías hacia esta comunidad autónoma nos llevó a diseñar un estudio de prevalencia en el área sanitaria de Lanzarote para conocer su incidencia real y hacer posible la detección de portadores en un grupo de riesgo como la población de mujeres gestantes, con el objetivo de proporcionar un correcto consejo genético y prevenir la aparición de formas severas de la enfermedad.

\section{MATERIAL Y MÉTODOS}

DISEÑO

Se ha realizado un estudio epidemiológico observacional transversal.

\section{PACIENTES}

De noviembre 2002 a septiembre 2004 se estudiaron de forma consecutiva las 2.436 mujeres gestantes que acudieron a la revisión periódica realizada en la consulta de Obstetricia durante el primer trimestre de la gestación en el Hospital General de Lanzarote.

\section{EXTRACCIÓN DE LA MUESTRA}

La extracción de la sangre se realizó en ayunas (entre las ocho y las nueve de la mañana) y se recogieron 5 mililitros de sangre en EDTA-K3 y 10 mililitros de sangre sin anticoagulante. Estas dos muestras se extraen de forma habitual en nuestro hospital a todas las mujeres en el protocolo del primer trimestre de la gestación por lo que no fue necesaria la extracción de muestras adicionales para el estudio.

\section{PROTOCOLO DE ESTUDIO DE LAS MUESTRAS}

Las muestras extraídas fueron manejadas según un esquema protocolizado (Fig. 1) que incluía la realización de un hemograma y un recuento de reticulocitos $\left(\right.$ Coulter $^{\circledR}$ ReticPrep $^{\mathrm{TM}}$ Reagent Kit) mediante el autoanalizador Coulter ${ }^{\circledR} \mathrm{LH}$ 750 y el examen de la morfología de la serie roja en una extensión de sangre teñida con May-Grümwald-Giemsa. El estudio del metabolismo férrico incluía sideremia mediante prueba colorimétrica basada en el método FerroZine sin desproteinización descrito por Persijn, transferrina sérica por test inmunoturbidimétrico basado en el principio de la prueba inmunológica de aglutinación y ferritina sérica por un test inmunoturbidimétrico basado en el principio de la prueba inmunológica de aglutinación con intensificación de la reacción por látex.

\section{ESTUDIOS BÁSICOS DE LA HEMOGLOBINA}

Todas las muestras fueron estudiadas para detectar la presencia de una hemoglobina variante realizando una electroforesis de hemoglobina en acetato de celulosa a $\mathrm{pH}$ alcalino $(\mathrm{pH}$ $=8,5)\left(\right.$ Sebia Electrophorese $\left(\right.$ Hydragel $^{\circledR} 15$ Hemoglobin $\left.(e)\right)$. Si se advertía la presencia de una hemoglobina variante se efectuaba posteriormente una electroforesis en gel de agar citrato a pH ácido $(\mathrm{pH}=6,0)$ Sebia Electrophorese (Hydragel ${ }^{\circledast} 15$ Acid Hemoglobin (e)) para confirmar la identificación de la hemoglobina encontrada y, en particular, para diferenciar la $\mathrm{HbS}$ de la $\mathrm{HbD}$ y la $\mathrm{HbE}$ de la $\mathrm{HbC}$.

Las muestras con microcitosis (volumen corpuscular medio < 80fL) y metabolismo férrico normal se seleccionaron para la cuantificación de la $\mathrm{HbA}_{2}$ y de la hemoglobina fetal $(\mathrm{HbF})$. La tasa de $\mathrm{HbA}_{2}$ se determinó por técnica de cromatografía de intercambio aniónico (DEAE celulosa) en microcolumna, mediante el equipo Beta-Thal $\mathrm{HbA}_{2}$ Quick Column suministrado por Helena Biosciences Laboratories y la $\mathrm{HbF}$ por inmunodifusión radial (Helen HbF Quiplate Kit, Helena Laboratories).

La $\beta$ talasemia heterocigota se consideró si el porcentaje de la $\mathrm{HbA}_{2}$ era superior a $3,5 \%$ por técnica de cromatografía en microcolumna (DEAE celulosa).

\section{ESTUDIOS CONFIRMATORIOS DE LA PRESENCIA DE UNA HEMOGLOBINA VARIANTE}

Los resultados patológicos en las electroforesis de celulosa a $\mathrm{pH}$ alcalino y/o en agar citrato a $\mathrm{pH}$ ácido, se incluían en el estudio de confirmación que incluía el isoelectroenfoque analí- 


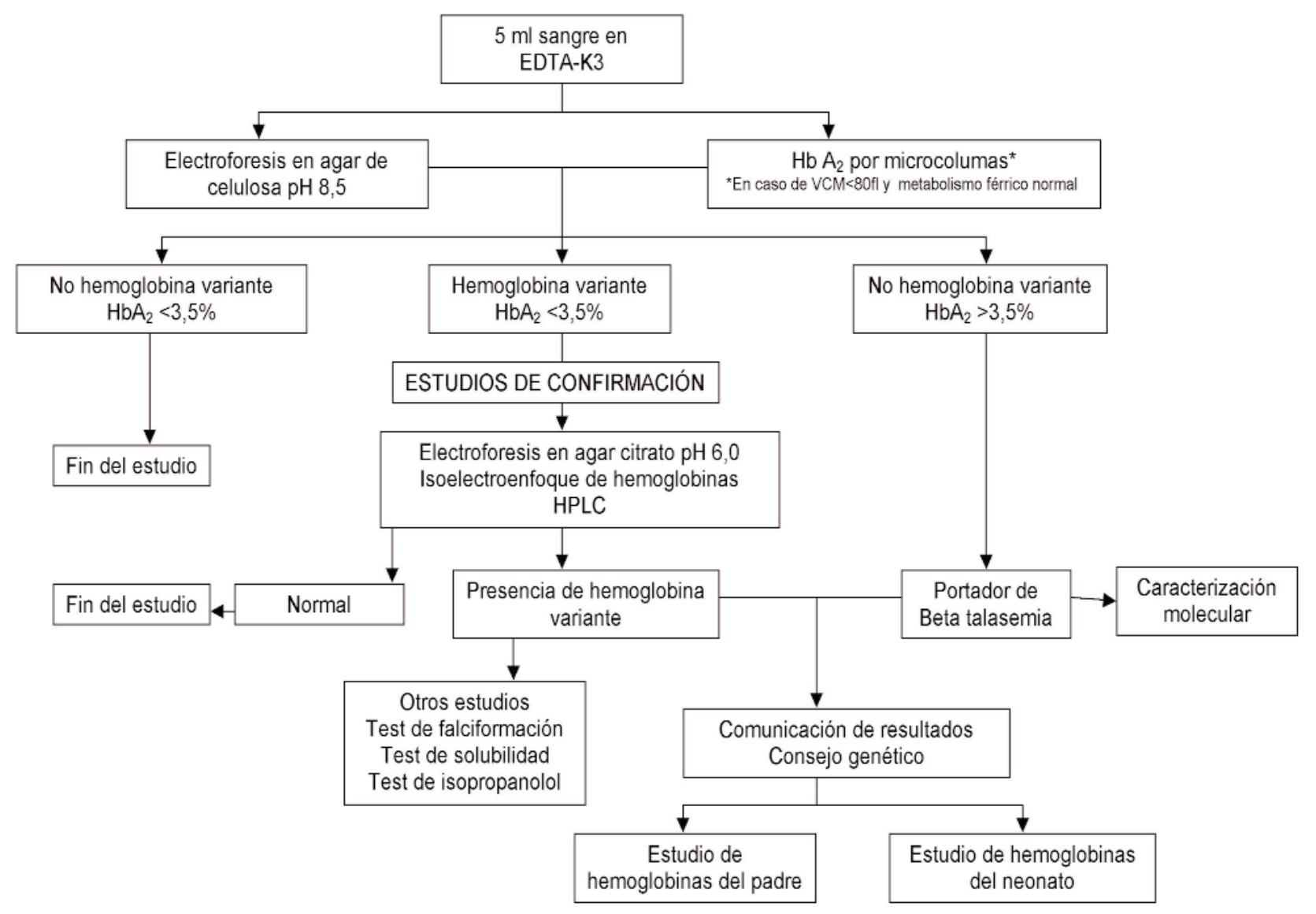

Fig. 1. Programa de detección de hemoglobinopatías estructurales y talasemias en Lanzarote.

tico de hemoglobinas en gel de poliacrilamida (PhastSystem ${ }^{\circledR}$, Pharmacia Biotech con geles de poliacrilamida PhastGel IEF media) y la cromatografía líquida de alta resolución (HPLC) (VARIANT $^{\text {TM }} \beta$ thalassemia Short Program, Bio Rad Laboratories con el kit $\beta$ thalassemia Short Program Reorder Pack).

La confirmación por estos métodos de una $\mathrm{HbS}$ conducía a completar el estudio con las pruebas de la falciformación y de la solubilidad de la hemoglobina y si el estudio sugería la presencia de una hemoglobina inestable se realizaron técnicas para determinar la estabilidad molecular de la hemoglobina mediante la prueba de la estabilidad de la hemoglobina frente al calor y frente al isopropanolol.

\section{ESTUDIOS MOLECULARES DE LAS TALASEMIAS}

La confirmación de la presencia de una $\beta$ talasemia, con una tasa de $\mathrm{HbA}_{2}$ superior al 3,5\%, y excluida la posibilidad de un doble heterocigocia hemoglobinopatía estructural $/ \beta$ talasemia determinaba la extracción del ADN leucocitario con el método de fenol-cloroformo con precipitación final del ADN con etanol frío para su estudio molecular con PCR.

El análisis molecular de las alteraciones del gen de la globina $\beta$ más frecuentes en nuestro ámbito geográfico como las mutaciones en los intrones IVS I- $1(\mathrm{G} \rightarrow \mathrm{A})$, IVS I-6 $(\mathrm{T} \rightarrow \mathrm{C})$ y IVS I-110 $(\mathrm{G} \rightarrow \mathrm{A})$ y de los codones CD39 $(\mathrm{C} \rightarrow \mathrm{T})$ y CD37 $(\mathrm{G} \rightarrow \mathrm{A})$ se caracterizó mediante sondas de hibridación marcadas con fluorógenos y PCR en tiempo real empleando el equipo termociclador Lightcycler-Roche. En los casos en que no se demostró alteración molecular con la PCR en tiempo real se empleó para el estudio molecular la reacción en cadena de la polimerasa Amplification Mutation System (PCR-ARMS ${ }^{\mathrm{TM}}$ ).

\section{COMUNICACIÓN DE LOS RESULTADOS}

Los resultados del estudio se entregaron a cada una de las mujeres gestantes portadoras de hemoglobinopatías en entrevista personal de forma confidencial informando de forma objetiva del significado clínico del estado de portador y aportando consejo genético.

El mismo esquema protocolizado de escrutinio de hemoglobinopatías se empleó con otros miembros de la familia de la mujer portadora de una hemoglobinopatía, con el padre y con el neonato. Los resultados se remitieron en un informe escrito a los individuos afectos y al médico de Atención Primaria incluyendo consejo genético. 
TABLA I

CARACTERÍSTICAS DE LAS 29 MUJERES GESTANTES PORTADORAS DE HEMOGLOBINOPATÍAS ESTRUCTURALES Y TALASEMIAS

\begin{tabular}{|c|c|c|c|c|c|c|c|c|c|}
\hline & \multirow{2}{*}{\multicolumn{2}{|c|}{$\begin{array}{c}\text { Total } \\
\text { Hemoglobinopatías } \\
(n=29)\end{array}$}} & \multirow{2}{*}{\multicolumn{2}{|c|}{$\begin{array}{c}\beta \text { Talasemias } \\
(n=6)\end{array}$}} & \multirow{2}{*}{\multicolumn{2}{|c|}{$\begin{array}{c}\text { Hemoglobinas } \\
\text { estructurales } \\
(n=23)\end{array}$}} & \multirow{3}{*}{$\begin{array}{c}\text { Hemo } \\
\text { Hemoglobina } \\
S \\
N\end{array}$} & \multirow{3}{*}{$\begin{array}{c}\text { binopatías estr } \\
\text { Hemoglobina } \\
\mathrm{C}\end{array}$} & \multirow{3}{*}{$\begin{array}{c}\text { cturales } \\
\begin{array}{c}\text { Hemoglobina D/ } \\
\text { otras }\end{array} \\
N\end{array}$} \\
\hline & & & & & & & & & \\
\hline & $N$ & $\%$ & $N$ & $\%$ & $N$ & $\%$ & & & \\
\hline \multicolumn{10}{|l|}{ Raza y/o etnia } \\
\hline Caucásica & 9 & $31,03 \%$ & 5 & $83,33 \%$ & 4 & $17,39 \%$ & 2 & 2 & - \\
\hline Negra/negroide & 14 & $48,27 \%$ & - & - & 14 & $60,86 \%$ & 9 & 4 & 1 \\
\hline Magrebí & 4 & $13,79 \%$ & 1 & $16,66 \%$ & 3 & $13,04 \%$ & 1 & - & 2 \\
\hline Indígena americano & 2 & $6,89 \%$ & - & - & 2 & $8,69 \%$ & 1 & 1 & - \\
\hline \multicolumn{10}{|l|}{ Localización geográfica } \\
\hline CANARIAS & 6 & $20,68 \%$ & 4 & $66,66 \%$ & 2 & $86,95 \%$ & - & 2 & - \\
\hline Lanzarote & \multicolumn{2}{|c|}{5} & \multicolumn{2}{|c|}{4} & \multicolumn{2}{|c|}{1} & - & 1 & - \\
\hline Fuerteventura & \multicolumn{2}{|c|}{1} & \multicolumn{2}{|c|}{-} & \multicolumn{2}{|c|}{1} & - & 1 & - \\
\hline ESPAÑA PENINSULAR & 2 & $6,89 \%$ & \multirow{2}{*}{\multicolumn{2}{|c|}{ - }} & 2 & $8,69 \%$ & 2 & - & - \\
\hline Cádiz & \multicolumn{2}{|r|}{$0,07 / 10$} & & & \multicolumn{2}{|r|}{0,0710} & 2 & - & - \\
\hline AFRICA & 8 & $57,58 \%$ & 1 & $16,66 \%$ & 7 & $30,43 \%$ & 5 & - & 2 \\
\hline Marruecos & \multicolumn{2}{|c|}{4} & 1 & & \multicolumn{2}{|c|}{3} & 1 & - & 2 \\
\hline Cabo Verde & \multirow{2}{*}{\multicolumn{2}{|c|}{$\begin{array}{l}1 \\
1\end{array}$}} & \multicolumn{2}{|c|}{ _ } & \multirow{2}{*}{\multicolumn{2}{|c|}{$\begin{array}{l}1 \\
1\end{array}$}} & 1 & - & - \\
\hline Guinea Bissau & & & & & & & 1 & - & - \\
\hline Nigeria & \multicolumn{2}{|c|}{1} & & & \multicolumn{2}{|c|}{1} & 1 & - & - \\
\hline Senegal & \multicolumn{2}{|c|}{1} & & & \multicolumn{2}{|c|}{1} & 1 & - & - \\
\hline AMERICA & 13 & $44,82 \%$ & 1 & $16,66 \%$ & 12 & $52,17 \%$ & 6 & 5 & 1 \\
\hline Argentina & \multirow{2}{*}{\multicolumn{2}{|c|}{$\begin{array}{l}1 \\
1\end{array}$}} & \multirow{2}{*}{\multicolumn{2}{|c|}{1}} & \multicolumn{2}{|c|}{-} & 1 & - & - \\
\hline Ecuador & & & & & & & - & - & - \\
\hline Belice & & & & & & & 1 & - & - \\
\hline Colombia & & & & & & & 1 & 3 & - \\
\hline Republica Dominicana & & & & & & & 1 & 2 & 1 \\
\hline Cuba & & & & & & & 2 & - & - \\
\hline Prevalencia (\%0) & & & & & & & 5,33 & 2,87 & 1,23 \\
\hline Edad media y rango (años) & & & & -35) & & & & $27,8(18-36)$ & \\
\hline
\end{tabular}

\section{RESULTADOS}

En el periodo de 23 meses incluidos entre el uno de noviembre de 2001 y el 30 de septiembre del 2003 se han analizado 2.436 muestras de sangre procedentes de sangre venosa de mujeres gestantes atendidas en la consulta de obstetricia del Hospital General de Lanzarote. El esquema de estudio propuesto ha detectado la presencia de una hemoglobinopatía en 29 muestras, veintitrés hemoglobinas variantes en el estudio electroforético de hemoglobinas a $\mathrm{pH}$ alcalino y seis $\beta$ talasemias heterocigotas.

En la Tabla I se presentan los datos de las mujeres gestantes portadoras de hemoglobinopatías estructurales y talasemias, las tasas de prevalencia global y por hemoglobinopatías, la distribución según el origen familiar y las mutaciones moleculares en las portadoras de $\beta$ talasemia .

La prevalencia global de hemoglobinopatías en Lanzarote representa un $11,90 \%$ distribuidas en una prevalencia de portadores de $\beta$ talasemia del $2,46 \%$ y del $9,44 \%$ o para las hemoglobinopatías estructurales heterocigotas.
El estudio del total de casos detectados muestra una incidencia global de hemoglobinopatías estructurales de 1 caso cada 106 mujeres analizadas y una incidencia de la forma heterocigota de la anemia falciforme de 1/187 gestantes.

Las muestras con una banda de migración anómala en la electroforesis de hemoglobina correspondían en trece casos a una hemoglobina $\mathrm{S}$ heterocigota lo que supone una prevalencia de rasgo falciforme de 5,33 casos por mil mujeres gestantes, siete fueron portadoras de una hemoglobina $\mathrm{C}$ con una prevalencia de $2,87 \%$, dos casos de una hemoglobina D con prevalencia del $0,82 \%$ y un caso de una hemoglobina inestable que no se pudo identificar al aplicar el algoritmo diagnóstico.

El origen familiar de diecinueve mujeres portadoras de hemoglobinopatías estructurales estaba en países no europeos, siete de origen africano y doce nacidas en países del centro y sur de América, mientras que las otras cuatro mujeres tenían origen en la España peninsular (Cádiz) en dos casos y dos eran canarias, una nativa de Lanzarote y otra procedente de Fuerteventura. Las dos gestantes canarias eran portadoras de 
una hemoglobina C, las dos con origen en Cádiz de una HbS y en las 19 inmigrantes extranjeras la hemoglobinopatía más frecuente fue la $\mathrm{HbS}$ encontrada en 11 gestantes $(54,5 \%$ origen africano y $45,5 \%$ iberoamericano), en cinco se detectó una $\mathrm{HbC}$ ( 3 colombianas y 2 dominicanas), en 2 marroquíes una $\mathrm{HbD}$ y en una mujer dominicana se encontró una hemoglobina inestable sin poder identificarla.

La prevalencia para el estado de portador de beta talasemia fue de 2,46 lo que supone una incidencia de $\beta$ talasemia heterocigota de 1 caso cada 406 mujeres incluidas en el estudio. Cuatro de las seis mujeres portadoras de $\beta$ talasemia eran de Lanzarote, y las otras dos venían de Argentina y Marruecos. La caracterización molecular mediante PCR a tiempo real con el sistema Lightcycler ha permitido la tipificación molecular en el $83,5 \%$ de las mujeres portadoras con cifras de HbA2 superiores al 3,5\%. La mutación más frecuente es la $\mathrm{CD}-39(\mathrm{C} \rightarrow \mathrm{T})$ que se ha detectado en el $66,6 \%$ (4 casos) de los portadores talasémicos, a la que le sigue la IVS-I-6 $(\mathrm{T} \rightarrow \mathrm{C})$ (Tabla I) detectada en 1 individuo $(16,6 \%)$ y la mutación $(\mathrm{T} \rightarrow \mathrm{A})$ en el codón 24 que se ha detectado sólo en una mujer procedente de Marruecos.

Los padres de los neonatos tuvieron en todos los casos una cifra de $\mathrm{HbA} 2$ inferior al 3,5\% y el estudio electroforético a distintos $\mathrm{pH}$ no demostró la presencia de una hemoglobina de migración anómala. La ausencia de hemoglobinas variantes se confirmó con las ténicas de isoelectroenfoque de hemoglobinas y HPLC.

\section{DISCUSIÓN}

Las hemoglobinopatías estructurales y las talasemias son enfermedades emergentes en España con una distribución mundial que varía de unas áreas geográficas a otras. Estudios epidemiológicos han demostrado que la distribución mundial de la malaria se superpone con la de las hemoglobinopatías más comunes aunque cada hemoglobinopatía se circunscribe relativamente a una región determinada (6).

El diseño del estudio para conocer la prevalencia de portadores de hemoglobinopatías existentes en la isla tuvo lugar para establecer una correcta evaluación de su coste y efectividad previamente a establecer de forma definitiva un programa de prevención y control de las hemoglobinopatías en Lanzarote.

La tasa de prevalencia de la enfermedad, el sustrato étnico de la población y el volumen de muestras a analizar son datos fundamentales a la hora de establecer cualquier programa de prevención y control de hemoglobinopatías (10). El registro sistemático de los portadores de estas alteraciones de la hemoglobina y de su origen geográfico puede también ayudarnos a mejorar el conocimiento de su epidemiología (11).

Los programas de cribado de hemoglobinopatías que se realizan de forma sistemática en neonatos actualmente en España tienen lugar en Extremadura (12) y en la comunidad de Madrid $(6,13)$ y en Galicia se ha emitido un informe favorable sobre la relación coste efectividad de un cribado selectivo en poblaciones con mayor riesgo de presentar hemoglobinopatías (10).

Los programas prospectivos de detección de portadores han sido propuestos por diferentes autores $(14,15)$ como un método efectivo en la prevención y control de las formas graves homocigotas y dobles heterocigotas de las hemoglobino- patías. En España existen diferentes escrutinios de hemoglobinopatías estructurales en sangre de cordón de neonatos, lo que plantea algunos problemas metodológicos para la detección de hemoglobinas variantes en recién nacidos prematuros y también en la cuantificación del porcentaje de $\mathrm{HbA}_{2}$ que suele ser indetectable en la muestra de sangre de cordón obtenida en los primeros días de vida y hace necesario, en algunos casos, una nueva extracción sanguínea a las dos o tres semanas del primer análisis para determinar adecuadamente el porcentaje de $\mathrm{HbA}_{2}$ y fetal (13). En nuestro caso y siguiendo la experiencia publicada por otros grupos $(16,17)$ hemos preferido un programa de cribado de portadores de hemoglobinopatías entre mujeres gestantes, ofrecer consejo genético y establecer el diagnóstico prenatal en los casos de parejas en riesgo de que el feto esté afectado por la forma homocigota o doble heterocigota combinada de hemoglobinopatías graves como la anemia falciforme.

La técnica para el escrutinio de las hemoglobinas variantes ha sido la electroforesis de hemoglobina en agar celulosa a medio alcalino lo que permite un cribado de las hemoglobinas estructurales a un menor coste que otros métodos más tecnificados y no disponibles de forma rutinaria en nuestro hospital. Asumiendo la experiencia en España de diversos grupos $(13,18)$ hemos incluido diversas técnicas de laboratorio de confirmación de la presencia de una hemoglobina variante. La presencia en una muestra de una banda de migración anómala en la electroforesis de hemoglobinas en agar celulosa a $\mathrm{pH}$ alcalino era suficiente para realizar una electroforesis en agar citrato a pH ácido, el isoelectroenfoque analítico en gel de poliacrilamida y la cromatografía líquida de alta resolución (HPLC) $(19,20)$ que mejora los resultados en sensibilidad y especificidad de las electroforesis de hemoglobinas con una mayor rapidez aunque con un costo económico mayor. La utilización restrictiva y secuencial de estas técnicas diagnósticas ha identificado las hemoglobinas variantes con un menor coste económico y una utilización más racional de nuestros recursos aunque el empleo de la HPLC en fase reversa ha sido propugnado como un método mejor en el despistaje de las hemoglobinopatías estructurales que los métodos electroforéticos (6).

Tomando en consideración las recomendaciones internacionales $(21)$ y la experiencia de otros grupos $(13,22)$ hemos incluido en el estudio de las hemoglobinopatías a todas las mujeres gestantes residentes en la isla sin seleccionar grupos de riesgo por origen geográfico, raza o pertenencia a grupos poblacionales con mayor prevalencia de hemoglobinopatías. La eficacia de un cribado dirigido a recién nacidos de poblaciones de mayor riesgo de padecer la enfermedad en función de la raza tiene algunas limitaciones al perder individuos en riesgo por la posibilidad de una clasificación incorrecta de la raza. Algunos artículos publicados por grupos europeos propugnan la importancia del cribado selectivo en población de alto riesgo de drepanocitosis en razón de la raza o etnia $(23,24)$ o en población inmigrante africana subsahariana $(18)$. Lanzarote tiene una proporción relativamente alta de población de etnias y razas con origen en zonas con alta prevalencia de hemoglobinopatías lo que haría al escrutinio universal rentable en el aspecto coste efectividad y los cribados selectivos deberían planificarse en zonas con poca población en riesgo y baja prevalencia de hemoglobinopatías $(21,25)$.

Un aspecto negativo de los programas de detección de enfermedades en gestantes es la ansiedad que puede aparecer 
en estas mujeres al conocer la posibilidad de transmitir una enfermedad genética grave al feto. Los resultados del escrutinio los comunicamos a la pareja en el menor tiempo posible para intentar no causar inquietud ni ansiedad en los padres.

La prevalencia de hemoglobinopatías en mujeres gestantes en el área sanitaria de Lanzarote fue del 11,90\%o, de las cuales un $2,46 \%$ correspondían a $\beta$ talasemias heterocigotas y $9,44 \%$ a hemoglobinopatías estructurales. Los resultados fueron algo superiores a los publicados en otras zonas de España utilizando sangre de cordón de neonatos $(4,6,13,18,26-28)$ y en donantes de sangre (9), cuyos resultados se muestran en la Tabla II. Todos estos trabajos realizados en zonas de España colonizadas durante su historia por pueblos de diferentes etnias, tienen en común una baja incidencia de hemoglobinopatías y talasemias en relación con otras series que incluyen individuos procedentes de países de la cuenca mediterránea, de Oriente próximo con población árabe o de distintas zonas de Africa.

Un estudio reciente realizado en Madrid (6) analizó una población con un número alto de neonatos hijos de inmigrantes y obtuvieron una incidencia de hemoglobinopatías de $1,17 \%$ ligeramente superior a lo obtenido en Lanzarote que también incluía población inmigrante.

La hemoglobina variante más detectada en Lanzarote ha sido la $\mathrm{HbS}$ (prevalente en el África subsahariana, India y península arábiga), lo que coincide con lo publicado por el resto de grupos españoles con la excepción de la zona del Delta del Ebro (27) que presenta una mayor frecuencia de una hemoglobina variante de la cadena gamma denominada $\mathrm{HbF}$ Catalonia. La siguiente hemoglobinopatía en frecuencia ha sido la hemoglobina $\mathrm{C}$ y la tercera la presencia de una $\beta$ talasemia, todas ellas responsables en su estado homocigoto o en su asociación (HbSC, HbS- $\beta$ talasemia) de enfermedades graves como la enfermedad drepanocítica que puede cursar con manifestaciones clínicas severas (19). El diagnóstico temprano es por tanto fundamental para poder establecer las medidas necesarias para reducir el riesgo de mortalidad precoz en estas enfermedades.

La prevalencia de $\beta$ talasemia heterocigota en las mujeres embazazadas de Lanzarote es algo inferior al 0,4\% establecida como promedio en España (3) y fue también más de tres veces inferior a la tasa de $0,81 \%$ (29) comunicada en mujeres gestantes en la provincia de Huelva.

El origen familiar de la mayoría de portadoras de $\beta$ talasemia $(66 \%)$ era la isla de Lanzarote, a diferencia de las mujeres con hemoglobinopatías estructurales que eran mayoritariamente población inmigrante de países africanos y americanos. La alteración molecular más prevalente en los portadores de $\beta$ talasemia fue la mutación del codon $39(\mathrm{C} \rightarrow \mathrm{T})$ que es también la más frecuente descrita en las regiones del Levante español y en las islas Baleares (3).

Los resultados obtenidos en este trabajo representan una aproximación a la situación real de las hemoglobinopatías en

TABLA II

ESTUDIOS DE PREVALENCIA DE LAS HEMOGLOBINOPATÍAS ESTRUCTURALES EN ESPAÑA

\begin{tabular}{|c|c|c|c|c|c|c|c|c|}
\hline Autor y año & $\begin{array}{l}\text { Localización } \\
\text { geográfica }\end{array}$ & $\begin{array}{l}\text { Tipo de } \\
\text { estudio }\end{array}$ & $\begin{array}{l}\text { Tamaño } \\
\text { muestral }\end{array}$ & $\begin{array}{l}\text { Prevalencia } \\
\text { global (\%o) }\end{array}$ & $\begin{array}{l}\text { Prevalenci } \\
\text { variante }\end{array}$ & $\begin{array}{l}\text { te } \mathrm{Hb} \\
\% \text { \%) }\end{array}$ & $\begin{array}{c}\text { Prevalencia de } \\
\text { HbS (\%o) }\end{array}$ & Población \\
\hline Baiget (5) (1981) & Barcelona & Transversal & 3.600 & 1,38 & $\begin{array}{l}1 \beta \\
4 \gamma\end{array}$ & $\begin{array}{l}0,27 \\
1,11\end{array}$ & 0,27 & Neonatos \\
\hline De Pablos (7) (1987) & ) Granada & Transversal & 9.977 & 1,25 & $\begin{array}{l}2 \alpha \\
8 \beta \\
3 \gamma\end{array}$ & $\begin{array}{l}0,2 \\
0,8 \\
1,11\end{array}$ & 0,18 & Neonatos \\
\hline $\begin{array}{l}\text { Martín Núñez (5) } \\
\text { (1989) }\end{array}$ & $\begin{array}{l}\text { Norte de } \\
\text { Cáceres }\end{array}$ & Transversal & 2.818 & 2,4 & $7 \beta$ & 0,27 & 0,10 & Neonatos \\
\hline Font (27) (1992) & $\begin{array}{l}\text { Delta del } \\
\text { río Ebro }\end{array}$ & Transversal & 2.206 & 2,2 & $\begin{array}{l}1 \beta \\
4 \gamma\end{array}$ & $\begin{array}{l}0,27 \\
1,11\end{array}$ & 0 & Neonatos \\
\hline $\begin{array}{l}\text { Malcorra (9) } \\
\text { (1994) }\end{array}$ & Gran Canaria & Transversal & 40.192 & 2,7 & $\begin{array}{c}100 \beta \\
6 \alpha\end{array}$ & $\begin{array}{l}0,27 \\
1,11\end{array}$ & 1,42 & Neonatos \\
\hline $\begin{array}{l}\text { Gómez-Chiari (5) } \\
\text { (2003) }\end{array}$ & Barcelona & Serie de casos & 22 & - & $22 \beta$ & 0,27 & - & Neonatos \\
\hline Dulin (13) (2003) & Madrid & Transversal & 29.253 & 3,3 & $\begin{array}{c}92 \beta \\
3 \gamma \\
2 \text { otras }\end{array}$ & $\begin{array}{l}3,06 \\
0,14 \\
0,14\end{array}$ & $\begin{array}{l}0,17 \text { homocigoto } \\
2,40 \text { heterocigoto }\end{array}$ & Neonatos \\
\hline Cabot (18) (1998) & Mataró & Transversal & 82 & 10,98 & $9 \beta$ & 10,98 & 1,22 homocigoto & Neonatos \\
\hline $\begin{array}{l}\text { González (6) } \\
\text { (2002) }\end{array}$ & Madrid & Transversal & 1.200 & 11,7 & $\begin{array}{c}11 \alpha \\
3 \beta \\
3 \gamma\end{array}$ & $\begin{array}{l}9,19 \\
2,51 \\
2,51\end{array}$ & 5 & Neonatos \\
\hline Risueño (28) (1995) & Cádiz & Transversal & 15.789 & 1,38 & $\begin{array}{c}23 \beta \\
1 \text { otras }\end{array}$ & $\begin{array}{c}1,33 \\
0,006\end{array}$ & 0,04 & Neonatos \\
\hline Calvo-Villas (2005) & Lanzarote & Transversal & 2.536 & 9,44 & $\begin{array}{c}22 \beta \\
\text { tb inestable }\end{array}$ & $\begin{array}{c}0,27 \\
0,4\end{array}$ & 5,33 & $\begin{array}{l}\text { Mujeres } \\
\text { gestantes }\end{array}$ \\
\hline
\end{tabular}


el área sanitaria de Lanzarote en la actualidad y puede servir como referencia en la comunidad Canaria y en provincias peninsulares que acojan un número alto de individuos procedentes de la inmigración. Los datos de prevalencia no pretenden ser el reflejo de la población autóctona con origen familiar en Lanzarote ya que nuestra isla es una mezcla de poblaciones de diferentes orígenes étnicos y la mayoría de las hemoglobinopatías estructurales detectadas $(82,6 \%)$ correspondían a población inmigrante de países en vías de desarrollo con altas tasas de prevalencia de estas patologías. Todas las mujeres estudiadas eran, no obstante, residentes en Lanzarote lo que a nuestro juicio da una imagen real de la situación actual de las hemoglobinopatías en el área sanitaria.

Un problema adicional con la población procedente de la inmigración es el desconocimiento del funcionamiento del sistema sanitario y la dificultad idiomática (30) en gran parte de estos individuos. La situación administrativa irregular de algunas mujeres no fue inconveniente para el escrutinio previo al parto, sin embargo el seguimiento posterior fue errático y no pudo garantizarse la efectividad del consejo genético en la totalidad de portadoras de hemoglobinopatías.

En nuestra opinión, la puesta en marcha de estudios prospectivos de prevalencia de hemoglobinopatías (estructurales y talasemias) en comunidades receptoras de población inmigrante permitiría conocer la prevalencia real en las diferentes poblaciones que las integran y emprender campañas de detección en grupos de riesgo como neonatos, escolares o mujeres gestantes para prevenir las formas severas o tratarlas de forma precoz.

\section{Bibliografía}

1. Weatherall DJ, Clegg JB. The thalassaemia syndromes. $3^{\mathrm{a}}$ ed. Oxford: Blackwell, 1981.

2. Baiget M. Structural hemoglobinopathies in Spain. Sangre (Barc) 1985; 30: 899-904.

3. Pérez Sirvent M, Moreno Miralles I, Bolufer Gilabert P. Alteraciones moleculares de las talasemias en España. Med Clin (Barc) 1999; 113: 789-794.

4. Oliva Berini E, López Andrés N, Segui Puntas M, Hernández Pons JL, Martí Camps F. Campaña para la detección de betatalasemia minor y prevención de la major en la isla de Menorca. Experiencia de dos años. Med Clin (Barc) 1990; 94: 81-84

5. Baiget M, Del Río E, Domenech M, Casals T, Bozzo M, Gimferrer E. Escrutinio de hemoglobinopatías en sangre de cordón umbilical. Biol Clin Hematol 1981; 3: 251-256.

6. González FA, Hojas R, Ropero P, Villegas A. Aumento de la incidencia de hemoglobinopatías estructurales y talasemias en España. Haematologica 2002; 87 (Supl. 1): 368-372.

7. De Pablos JM. Hemoglobinopatías estructurales en España. Biol Cin Hematol 1988; 10: 5-15.

8. Malcorra JJ, Balda MI, Campo C, Mataix R, Molero T, Castro E. Hemoglobinopatías y talasemias en la colonia hindú afincada en las islas canarias. Sangre 1993; 38: 342.

9. Malcorra Azpiazu JJ. Hemoglobinopatías estructurales en Canarias. Haematologica 1998; 83 (Supl. 1): 413-419.

10. Ruano Raviña A, Jato Díaz. Cribado neonatal de hemoglobinopatías. Santiago de Compostela: Servicio Galego de Saúde, Axencia de Avalación de Tecnoloxias Sanitarias de Galicia, avalia-t; 2004. Serie Avaliación de tecnologías. Informes de evaluación INF2004/004.

11. Hernández JA, Bosch MA, Sauca G, Rovira JM, Clapés V, del Río N, et al. Hematología e inmigración. Impacto de la inmigración africana subsahariana en la práctica hematológica. Haematologica 2002; 87 (Supl. 1): 373-377.

12. Dirección General de la Salud Pública de Extremadura. Programa de detección precoz de minusvalías, 2003.

13. Dulin Inigueza E, Cantalejo López MA, Cela de Julián ME, Galaron García P. Detección precoz neonatal de anemia falciforme y otras hemoglobinopatías en la Comunidad Autónoma de Madrid. Estudio piloto. An Pediatr (Barc) 2003; 58: 146-155.

14. Gessner BD, Teutsch SM, Shaffer PA. A cost-effectiveness evaluation of newborn hemoglobinopathy screening from the perspective of state health care systems. Early Hum Dev 1996; 45: 257-275.

15. Cronin EK, Normand C, Henthorn JS, Hickman M, Davies SC. Costing model for neonatal screening and diagnosis of haemoglobinopathies. Arch Dis Child Fetal Neonatal Ed 1998; 79: F161-F167.

16. Hendy J. Preventation of thalassemia in Australia. Southeast Asian J
Trop Med Public Health 1999; 30 (Supl. 2): 94-96.

17. Sanchaisuriya K, Fucharoen S, Fucharoen G, Ratanasiri T, Sanchaisuriya $\mathrm{P}$, Changtrakul Y, et al. A reliable screening protocol for thalassemia and hemoglobinopathies in pregnancy: an alternative approach to electronic blood cell counting. Am J Clin Pathol 2005; 123: 113-118.

18. Cabot Dalmau, Casado Toda M, Barberán Pérez J, Roqueta Sureda M, Martorell Aymerich Q, Bosch Llobet A, et al. Screening neonatal de drepanocitosis en el Consorci Sanitari de Mataró. Justificación y primeros resultados. An Esp Pediatr 1998; 49: 157-160.

19. Eastman JW, Wong R, Liao CL, Morales DR. Automated HPLC screening of newborns for sickle cell anemia and other hemoglobinopathies. Clin Chem 1996; 42: 704-710.

20. Davies SC, Cronin E, Gill M, Greengross P, Hickman M, Normand C. Screening for sickle cell disease and thalassaemia: a systematic review with supplementary research. Health Technol Assess 2000; 4: 1-99.

21. NIH Consens Statement. Newwborn screening for sickle cell disease and other hemoglobinopathies. JAMA 1987; 258.

22. Hansen V, Gulbis B, Humblet P, Cotton F, Vertongen F. Screening for hemoglobinopathies in medical practice: audit of gynecologists, pediatricians and generalists in Brussels. Rev Med Brux 2001; 22: 67 72 .

23. Ducrocq R, Benkerrou M, Brahimi L, Belloy M, Briard ML, Vilmer E, et al. Neonatal screening for sickle cell anemia: evaluation of a five-year experience in an area of northern Paris. Arch Pediatr 2001; 8: 474-80.

24. Gómez-Chiari M, Tusell Puigbert J, Ortega Aramburu J. Drepanocitosis: experiencia en un centro. An Pediatr (Barc) 2003; 58: 95-99.

25. Joiner $\mathrm{CH}$. Universal newborn screening for hemoglobinopathies. J Pediatr 2000; 136: 145-146.

26. Martín Núñez G, Ramos Fernández de Soria R, Fernández Galán MA, Sánchez Gil F, Cuesta P, Martín Borregón J, et al. Campaña para la detección de hemoglobinopatías y talasemias en la población escolar del norte de Extremadura Sangre 1995; 40: 459-464.

27. Font Ferré LI. Estudio de las hemoglobinopatías en la zona del delta del ría Ebro. Biol Clin Hematol 1993; 15: 87-96.

28. Risueño CE, Castro JM, Villegas A, Muñoz JA. Las hemoglobinopatías en la bahía de Cádiz. Sangre 1995; 40: 233-234.

29. Benito A, Villegas A, Pérez-Cano R, Bernal R. Beta-thalassaemia in south-western Spain: high frequency of $\mathrm{G} \rightarrow \mathrm{A}$ (IVS I-1) mutation. Br J Haematol 1996; 92: 336-338.

30. Berra S, Elorza Ricart JM, Bartomeu NH, Serra V, Rajmil L. Necesidades en salud y utilización de los servicios sanitarios en la población inmigrante en Cataluña. Revisión exhaustiva de la literatura científica. Barcelona: Agencia de Evaluació de Tecnología et Recerca Médiques. CatSalud. Departament de Sanitat i Seguretat Social. Generalitat de Catalunya; 2004. 